\title{
Two modes of propagating waves in sunspots
}

\author{
D. Y. Kolobov and N. I. Kobanov \\ Institute of Solar-Terrestrial Physics, P.O. Box 291, 664033, Irkutsk, Russia \\ email: kolobov@iszf.irk.ru
}

\begin{abstract}
The observations of sunspot chromosphere are presented. The authors suggest the existence of two modes of propagating waves at the chromospheric level. The connection between these modes and magnetic field topology can be inferred from the analysis of mode propagation velocity and spatial localization. Two hypotheses are tested to explain the phenomenon: "Visual pattern" and "Trans-sunspot wave".
\end{abstract}

Keywords. Sun: sunspots, Sun: oscillations, Sun: chromosphere, Sun: photosphere

\section{Introduction}

Since the discovery of propagating waves in the chromosphere of sunspots (Beckers, J. M. \& Tallant, P. E., Giovanelli (1972), Zirin \& Stein (1972)), the relationship between three-minute running waves in the umbra (RUW) and running penumbral waves (RPW) has been a most intriguing question. Two possible scenarios the "trans-sunspot wave" and the "visual pattern" are most frequently considered. Some researchers (Alissandrakis, Georgakilas \& Dialetis (1992), Tziotziou, Tsiropoula, Mein, et al. (2006)) are of the opinion that an RPW is the direct continuation of an RUW ("trans-sunspot wave"). Others consider them to be independent, although they do not rule out the possibility of a common subphotospheric source (Christopoulou, Georgakilas \& Koutchmy). Recently, the assumption (Rouppe van der Voort, et al. (2003), Bogdan \& Judge (2006)) that running waves in the chromosphere of sunspots are an illusion is more often expressed ("visual pattern"). According to this version, the effect occurs thanks to the fact that waves propagating from below along shorter vertical magnetic tubes reach an observable height level earlier than waves propagating along more oblique and consequently longer tubes. Meanwhile, one gets the impression that the wave front propagates horizontally from the umbra to the penumbra. This version requires an extensive sub photospheric source a role that could be played by a convection cell. Here we analyse which of the scenario is in agreement with observations.

The observations were carried out on the Horizontal Solar Telescope at the Sayan Solar Observatory. The method used allows us to measure the longitudinal magnetic field in the photosphere (Fe I $6569 \AA, \mathrm{g}=1.4$ ), intensity and line-of-sight (LOS) velocity (H $\alpha$ ) oscillations simultaneously. The power spectra were derived by applying a wavelet transformation. We used methods of frequency filtration to study the behaviour of propagating sunspot waves. More information can be found in Kobanov, Makarchik \& Sklyar (2003).

\section{Space-time localisation of different modes}

The results achieved with a help of frequency filtration technique are shown below. The spots NOAA 791, NOAA 794, NOAA 657 are different in size but have more or less rounded and homogeneous umbrae. The space-time distribution of power of the filtered modes is presented in figure 1 . The main power of three-minute oscillations is 

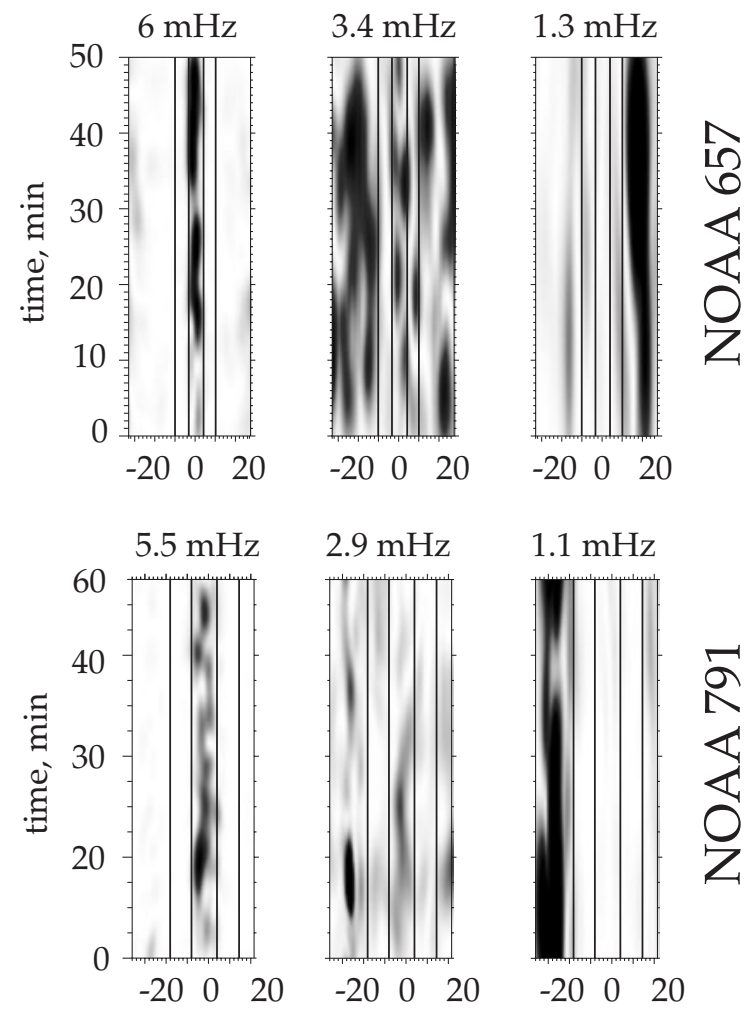

\section{$1.1 \mathrm{mHz}$}
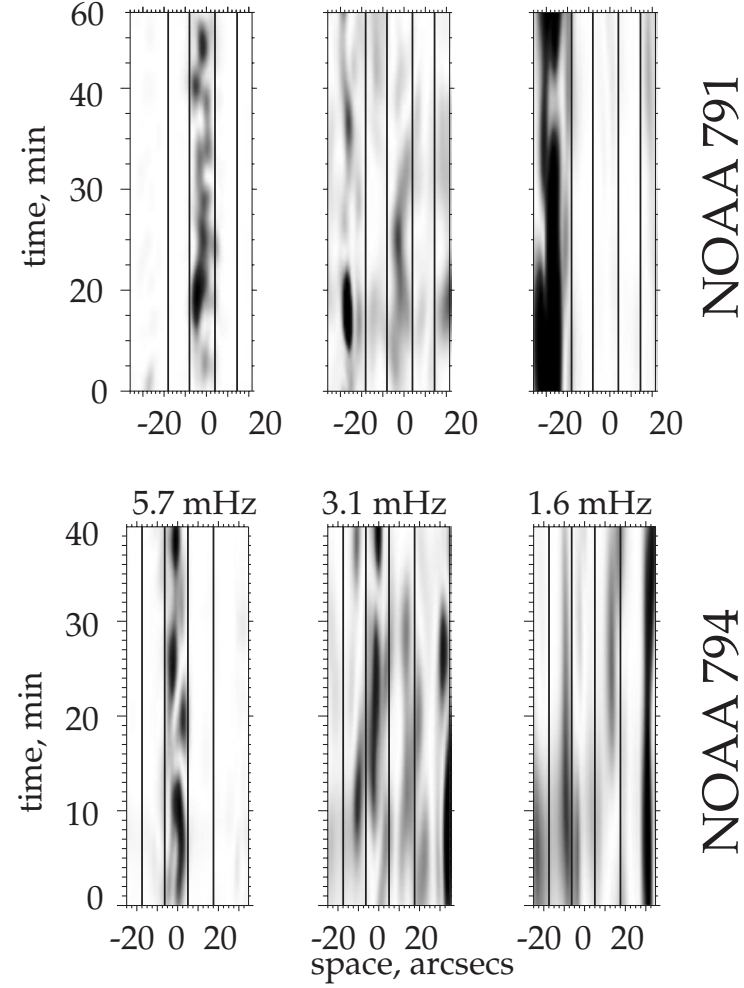

Figure 1. Space-time diagrams of LOS velocity power of different frequency, filtered with wavelet (black - maximum power)

concentrated in the centre of the umbra. Five-minute oscillations are localised in the penumbra, while long-term modes dominate in the penumbra and superpenumbra. Attention is drawn to the fact that the moments of occurrence of maximum power do not coincide. This is evidence of an absence of a direct mechanical link between modes. If we were observing a process of simple frequency transformation as the wave propagates, such moments would coincide. 


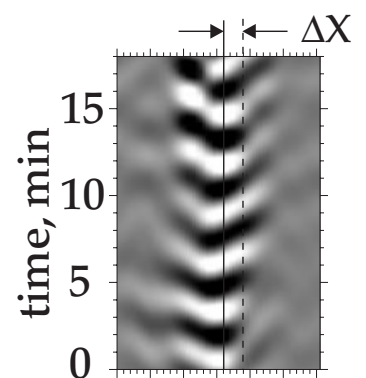

$\begin{array}{lll}-15 & 0 & 15\end{array}$

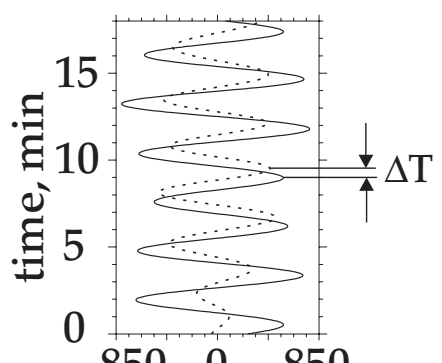

$\begin{array}{lll}-850 & 0 & 850\end{array}$

space, arcsecs LOS velocity, $\mathrm{m} / \mathrm{s}$

$\Delta \mathrm{X}=3$ arcsecs, $\Delta \mathrm{T}=35 \mathrm{~s}$, Propagation velocity
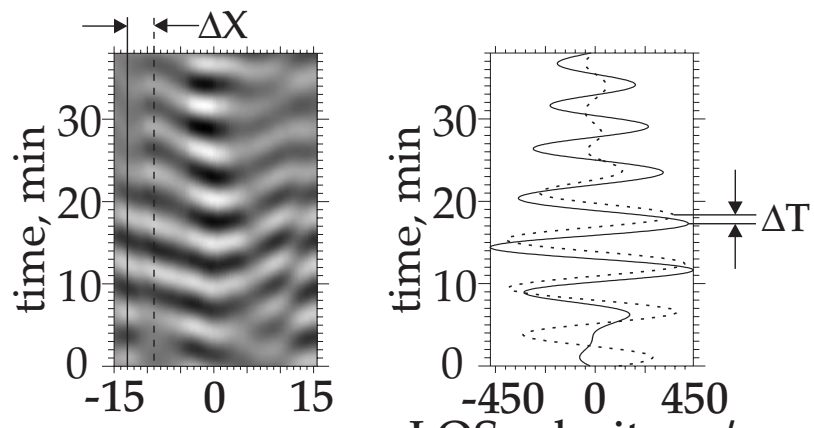

\section{$2.9 \mathrm{mHz}$ \\ filtered mode}

LOS velocity, $\mathrm{m} / \mathrm{s}$

\section{$\Delta \mathrm{X}=4 \operatorname{arcsecs}, \Delta \mathrm{T}=70 \mathrm{~s}$, Propagation velocity $41 \mathrm{k} \mathrm{m} / \mathrm{s}$}

Figure 2. Space-time diagrams of LOS velocity (filtered with wavelet). $\mathrm{H} \alpha$ line, NOAA 791

\section{Filtered space-time velocity diagrams}

A quasirandom distribution of different modes in time can serve as a reason why the resulting velocity of movement of the wave front, determined with the help of unfiltered diagrams, strongly depends on the choice of time interval. Apparently, this is not the case when only one filtered mode is under investigation. In our opinion, it is more convenient to measure propagation velocity with the help of chevrons (Kobanov, Kolobov \& Makarchik (2006), Kobanov \& Makarchik (2004)) that are clearly visible in the half-tone LOS velocity diagrams plotted for the main frequency modes 2.9 and $5.5 \mathrm{mHz}$ (figure 2). Velocity can be determined both according to the chevron slope and also with the help of LOS velocity phase delay measured at different spatial points. The chevron wings in these figures look quite linear. This means that the phase velocity remains almost unchanged in the process of wave propagation.

\section{Visual pattern scenario}

The observed properties of waves with different periods suggest that the "trans-sunspot" scenario can not explain their nature. Below we consider a brief test of another scenario: "visual pattern" (figure 3), and how phase lags, measured by different authors, correspond to $h$, the depth at which magnetic field lines start to incline at 30 degrees. $h=6.4 \times r \times V_{\text {snd }} /$ Vphase, where $V_{\text {snd }}$ is the sound speed of the upward propagating 
Visual pattern scenario to explain travelling waves

a)
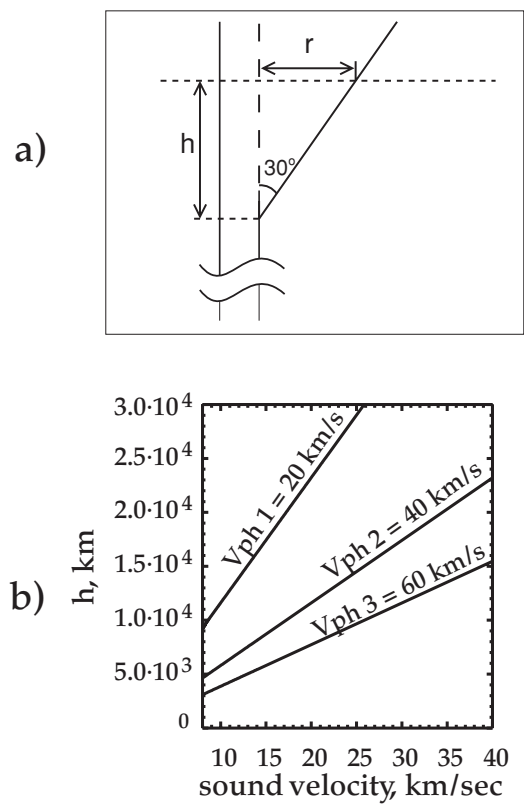

$\mathrm{r}$ - sunspot umbra radius

$\mathrm{h}$ - length of central trajectory

to produce measuredlag

$\mathrm{h} \cdot \mathrm{Vsnd}=6.4 \cdot \mathrm{dT}$

$\mathrm{dT}=\mathrm{r} /$ Vphase - phase lag

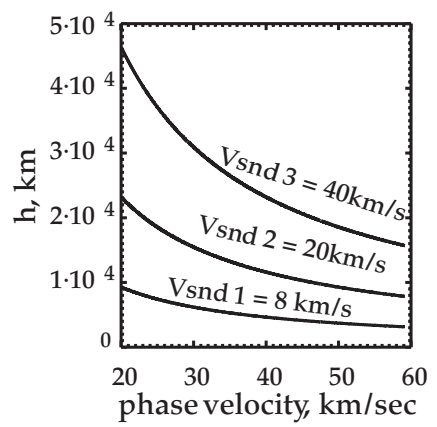

Figure 3. a) sketch to illustrate geometry of the produced lag b) $h$ as a function of: $V_{\text {sound }}$ (left panel) and $V_{\text {phase }}$ (right panel)

wave changing from subphotosperic values to chromospheric ones. $V_{\text {phase }}$ is the measured phase speed. For example, taking the $V_{\text {phase }}=20 \mathrm{~km} / \mathrm{s}$ (Tziotziou, Tsiropoula, Mein, et al. (2006)), the $h$ will be around $10000-30000 \mathrm{~km}$. The phase speed of $40-60 \mathrm{~km} / \mathrm{s}$ (Kobanov, Kolobov \& Makarchik (2006)) gives more plausible values of $h$ less or around $10000 \mathrm{~km} . V_{\text {snd }}$ is taken from different models of the solar atmosphere (see ChristensenDalsgaard, et al. (1996), Lites \& Skumanich (1982), Staude J. (1981)). $r$ is taken to be $4000 \mathrm{~km}$.

\section{Altitude inversion of localisation of three-minute oscillations}

It looks like the "visual pattern scenario" is in agreement with observed properties of travelling waves. But in the case of direct propagation of oscillation from photosphere to chromosphere, the three-minute mode would exist at both levels, though possibly with less amplitude. At least they would reveal amplitudes not less than those of the surrounding photosphere. The observations show a completely opposite situation: the maximum power of three-minute oscillations in the chromosphere corresponds to their minimum in the photosphere. The latter makes it unclear how the oscillations appear at the chromospheric level.

\section{Conclusions}

Methods of frequency filtration provide illustrative material having greater expressiveness and adequacy in estimation of the parameters of the travelling waves.

The decrease in frequency and propagation velocity observed by other authors can be explained by the combined action of different frequency modes. As was shown above, the spatial localisation of modes corresponds with this supposition. Note that the measured 

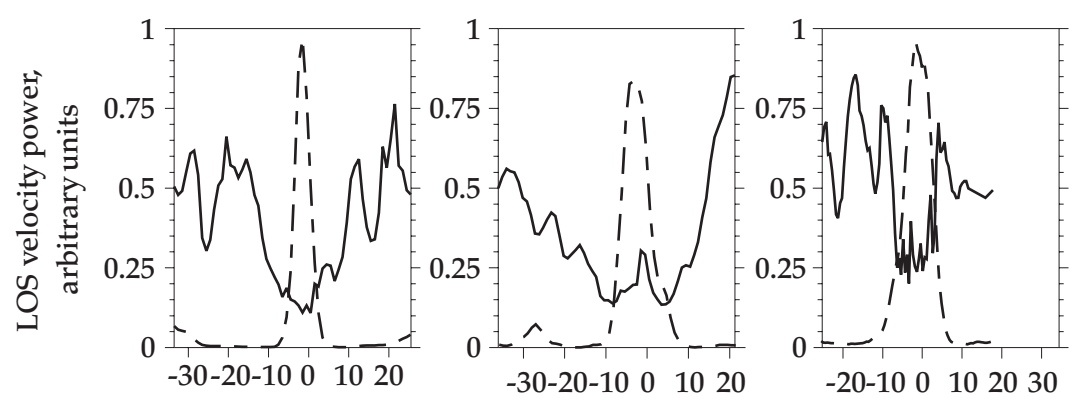

Figure 4. Average profile of LOS velocity at the photospheric level (filtered three-minute mode). Fe I 6569§ line, sunspots: NOAA 657 (left panel), NOAA 791 (middle panel), NOAA 794 (right panel)

horizontal propagation velocities are $2-3$ times higher than those measured by other authors $(40-70 \mathrm{~km} / \mathrm{s}$ for RUW, and $30-70 \mathrm{~km} / \mathrm{s}$ for RPW).

The localisation of different modes and moments of occurrence of its maximum power do not coincide. The trans-sunspot wave scenario does not explain the observable properties of umbral three-minute oscillations. The "Visual pattern" scenario looks more preferable, but the effect of altitude inversion raises new questions about the origin of three-minute oscillations.

\section{Acknowledgements}

The work is supported in part by the RFBR grant 05-02-16325 and RAS program No. 16 .

\section{References}

Alissandrakis, C. E., Georgakilas, A. A. \& Dialetis, D. 1992, Sol. Phys. 138, 93

Beckers, J. M. \& Tallant, P. E. 1969, Sol. Phys. 7, 351

Bogdan, T.J. and Judge, P.G. 2006, Phil. Trans. R. Soc. London A. 364, 313

Christensen-Dalsgaard J., et al. 1996, Science 272, 1286

Christopoulou, E.B., Georgakilas, A.A. \& Koutchmy, S. 2000, A\&A 354, 305

Giovanelli, R. G. 1972, Sol. Phys. 27, 71

Kobanov N.I., Kolobov D.Y. \& Makarchik D.V. 2006, Sol. Phys. 238, 231

Kobanov N.I. \& Makarchik D.V. 2004, A\&GA 424, 671

Kobanov N.I., Makarchik D.V. \& Sklyar A.A. 2003, Sol. Phys. 217, 53

Lites B.W. \& Skumanich A. 1982, ApJ 49, 293

Rouppe van der Voort, L. H. M., et al. 2003, A\&A 403, 277

Staude J. 1981, A\&SA 100, 284

Tziotziou K., Tsiropoula G., Mein N., et al. 2006, A\&A 456, 689

Zirin, H. \& Stein, A. 1972, ApJ 1978, L85 